\title{
The COPD assessment test (CAT): response to pulmonary rehabilitation. A multicentre, prospective study
}

\author{
James W Dodd, ${ }^{1}$ Lauren Hogg, ${ }^{2}$ Jane Nolan, ${ }^{1}$ Helen Jefford, ${ }^{3}$ Amy Grant, ${ }^{4}$ \\ Victoria M Lord, ${ }_{1}^{5}$ Christine Falzon, ${ }^{6}$ Rachel Garrod, ${ }^{7}$ Cassandra Lee, ${ }^{8}$ Michael I Polkey, \\ Paul W Jones, ${ }^{1}$ William D-C Man, ${ }^{5}$ Nicholas S Hopkinson ${ }^{5}$
}

\begin{abstract}
${ }^{1}$ St George's Hospital NHS
Trust, London, UK

${ }^{2}$ Guy's and St Thomas'

Foundation NHS Trust, London, UK

${ }^{3}$ Greenwich Primary Care Trust, London, UK

${ }^{4}$ Croydon Primary Care Trust, London, UK

${ }^{5}$ The NIHR Respiratory

Biomedical Research Unit, Royal Brompton \& Harefield NHS

Foundation Trust and Imperial College, London UK

${ }^{6}$ Central London Community Healthcare, London, UK

'King's College Hospital NHS Foundation Trust, London, UK ${ }^{8}$ Imperial College Healthcare NHS Trust, UK
\end{abstract}

\section{Correspondence to} Nicholas Hopkinson, The NIHR Respiratory Biomedical Research Unit, Royal Brompton \& Harefield NHS Foundation Trust and Imperial College, Royal Brompton Hospital, Fulham Rd, London SW3 6NP, UK; n.hopkinson@ic.ac.uk

Received 29 November 2010 Accepted 16 February 2011 Published Online First 12 March 2011

\section{ABSTRACT}

Background The COPD (chronic obstructive pulmonary disease) assessment test (CAT) is a recently introduced, simple to use patient-completed quality of life instrument that contains eight questions covering the impact of symptoms in COPD. It is not known how the CAT score performs in the context of clinical pulmonary rehabilitation (PR) programmes or what the minimum clinically important difference is.

Methods The introduction of the CAT score as an outcome measure was prospectively studied by PR programmes across London. It was used alongside other measures including the St George's Respiratory Questionnaire, the Chronic Respiratory Disease Questionnaire, the Clinical COPD Questionnaire, the Hospital Anxiety and Depression score, the Medical Research Council (MRC) dyspnoea score and a range of different walking tests. Patients completed a 5-point anchor question used to assess overall response to PR from 'I feel much better' to 'I feel much worse'.

Results Data were available for 261 patients with COPD participating in seven programmes: mean (SD) age 69.0 (9.0) years, forced expiratory volume in $1 \mathrm{~s}\left(\mathrm{FEV}_{1}\right) 51.1$ (18.7) \% predicted, MRC score 3.2 (1.0). Mean change in CAT score after PR was 2.9 (5.6) points, improving by 3.8 (6.1) points in those scoring 'much better' $(n=162)$, and by $1.3(4.5)$ in those who felt 'a little better' $(n=88)$ $(p=0.002)$. Only eight individuals reported no difference after PR and three reported feeling 'a little worse', so comparison with these smaller groups was not possible. Conclusion The CAT score is simple to implement as an outcome measure, it improves in response to PR and can distinguish categories of response.

\section{INTRODUCTION}

In patients with chronic obstructive pulmonary disease (COPD), pulmonary rehabilitation (PR) can reduce symptoms, improve activity, restore independent function and reduce healthcare utilisation. $^{1-3}$ Validated tools including the St George's Respiratory Questionnaire (SGRQ), ${ }^{4}$ the Chronic Respiratory Questionnaire (CRQ) ${ }^{5}$ and the Clinical COPD Questionnaire (CCQ) ${ }^{6}$ have been used to assess the impact of PR on health-related quality of life. However, current health-related quality of life questionnaires are complex, timeconsuming to complete and may require specialist software or licences to use, which limits their applicability in routine practice.

\section{Key messages}

What is the key question?

- Does the COPD assessment test (CAT), a new health status measure for the condition, show a response to pulmonary rehabilitation?

What is the bottom line?

- A fall (improvement) in the score occurs following pulmonary rehabilitation which was larger in those who felt 'much better' rather than in those who felt only 'a little better'.

\section{Why read on?}

- The article compares response to the CAT, which is simple to implement and score, with response to other outcome measures currently in use for pulmonary rehabilitation.

The COPD assessment test (CAT) ${ }^{7}$ was developed as a short, simple instrument for quantifying the symptom burden of COPD in routine practice to aid health status assessment and facilitate communication between patient and healthcare professionals. It consists of eight items, each presented as a semantic 6-point differential scale, providing a score out of 40 indicating the impact of the disease. It is completed by the patient and the result is immediately available without the need for any calculation, apart from summing the scores on individual items. Scores of 0-10, 11-20, 21-30 and 31-40 represent mild, moderate, severe or very severe clinical impact. ${ }^{8}$

The CAT was derived from 21 candidate items identified through qualitative research with patients with COPD from three prospective international studies (Europe and the USA, $n=1503$ ). Psychometric and Rasch analyses identified eight items fitting a unidimensional model to form the CAT, with high internal consistency (Cronbach $\alpha$ was 0.88$)$. Intraclass correlation coefficient was 0.8 for test-re-test in stable patients and CAT score correlated well with the COPD-specific version of the SGRO $(r=0.80)$. There are data to show that it distinguishes stable from exacerbating patients, ${ }^{7}$ but as yet no data on how it responds to PR or any other intervention.

PR programmes routinely use various methods to collect data about patients' baseline characteristics 
including health status and to assess their response to rehabilitation. The adoption of the CAT as an outcome measure by a network of pulmonary rehabilitation centres around London provided the opportunity to evaluate its responsiveness relative to other outcome measures used in rehabilitation studies and explore the use of an anchor questionnaire to address the minimum clinically important difference for the CAT.

\section{METHODS}

We performed a multicentre, prospective study of response to $\mathrm{PR}$ in patients with a clinical diagnosis of COPD. The study was approved by the Riverside Research Ethics Committee and recorded on an international trials register: ISRCTN51185878. Participating sites were located throughout London in both primary and secondary care settings. Patients were referred into programmes by a medical practitioner. All programmes included a mixture of aerobic and strength training and a mixture of supervised sessions and unsupervised home exercise, usually two supervised and one or more home sessions per week, with a duration of 8 weeks. Initial exercise prescription was based on the outcome of a baseline walking test, and workloads were increased through the programme as tolerated. Programmes were multidisciplinary, with an educational component covering issues including exercise, medication use, diet and coping strategies. Data were collected between January and August 2010 and recorded at enrolment and completion of the programmes.

The primary objective of the study was to establish the change in CAT score occurring in response to PR, relating this to an anchor question to explore CAT scores that may be indicative of the minimum clinically important difference (MCID). Response to the anchor question used to assess overall response to rehabilitation, 'How do you feel your overall condition has changed after rehabilitation?' was scored; 1, 'I feel much better'; 2, 'I feel a little better'; 3, 'I feel no different'; 4, 'I feel a little worse'; 5, 'I feel much worse'. The anchor question was asked at the end of the final assessment session.

Secondary objectives were to compare baseline CAT and change in CAT score with other health status and functional parameters measured in rehabilitation and to measure effect size of the tests being evaluated. The programmes in this study used a range of different baseline measures and outcomes, and the use of some outcomes varied between sites within programmes, so that the sample size for comparing the CAT with other measures was variable.

\section{Description of other comparator outcome measures used}

The $\mathrm{CCQ}^{6}$ is a self-administered questionnaire developed to measure clinical control in patients with COPD. It includes 10 items; each response is graded 1-6, with a higher score indicating worse health status. Cronbach $\alpha$ is high (0.91). Significant correlations have been demonstrated between the CCO total score and domains of the SGRO $(\mathrm{r}=0.67-0.72)$. In patients with COPD, the correlation between the CCQ and forced expiratory volume in $1 \mathrm{~s}\left(\mathrm{FEV}_{1}\right) \%$ predicted was $\mathrm{r}=-0.49$. Test-re-test reliability is high (intraclass coefficient $=0.94$ ). The minimum clinically important difference of the CCQ is $0.4 .^{9}$

The SGRQ ${ }^{4}$ consists of 50 items with 76 weighted responses. It was developed and validated in both asthma and COPD. It is completed by the patient by hand, but requires a computer to score it. Scores are calculated for three domains: symptoms, activity and impacts (psychosocial), as well as a total score. Psychometric testing has demonstrated its repeatability, reliability and validity. Scores range from 0 to 100 , with a higher score indicating worse health status. A minimum change in score of four units was established as clinically relevant after patient and clinician testing. The SGRO correlates significantly with other measures of disease activity such as cough, dyspnoea, 6 min walk test (6MWT) and $\mathrm{FEV}_{1}$, as well as other measures of general health such as the SIP (sickness impact profile) and SF36 (short form 36).

The $\mathrm{CRO}^{5}$ is a self-reported questionnaire developed to determine the effect of treatment on quality of life in clinical trials. It consists of four dimensions: dyspnoea, fatigue, emotional function and the patient's feeling of control over the disease (mastery). Reproducibility, tested by repeated administration to patients in a stable condition, has been excellent: the coefficient of variation was $<12 \%$ for all four dimensions. An MCID has been determined as 0.5 for each of the mean domain scores of the CRO. ${ }^{10}$

The incremental shuttle walking test ISWT (ISWT) uses a $10 \mathrm{~m}$ course and the walking speed is externally paced by signals from an audio cassette or CD. The patient is required to walk between two cones in time to a set of auditory beeps. ${ }^{11}$ The patient walks for as long as they can until they are either too breathless or can no longer keep up with the beeps, at which time the test ends. The number of shuttles (laps between the cones) is recorded. The results of the ISWT can be used to prescribe the intensity of walking exercise. Following PR in patients with COPD, an improvement of $47.5 \mathrm{~m}$ in ISWT corresponded to a patient perception that their exercise performance was 'slightly better', and an improvement of $78.7 \mathrm{~m}$ corresponded with 'better'. 12

The endurance shuttle walk test $(E S W T)^{13}$ is a standardised field test for the assessment of endurance capacity in patients with chronic lung disease. The test was developed as an adjunct to the ISWT so that together they form a practical method of assessing both functional and endurance exercise capacity using the same $10 \mathrm{~m}$ shuttle course.

The 6MWT is a self paced test that measures the distance that a patient can quickly walk in a period of 6 min (the 6MWD). ${ }^{14}$ Verbal instructions are standardised. Optimal reference equations from healthy population-based samples using standardised 6MWT methods have recently become available, and a walking distance in excess of $500 \mathrm{~m}$ is typical for patients of an age comparable with those entering PR. ${ }^{15}$

\section{Statistics and data analysis}

The data were anonymised at each site and collated centrally for analysis using SPSS v18. Paired t tests were used for comparison of CAT score before and after rehabilitation sessions. Univariate analysis of variance was used for each anchor question response. Correlations between change in CAT and other measures of health status and disease severity were calculated using Pearson correlations and linear regression tools; significantly skewed data were log transformed before analysis. Effect size was calculated as the mean difference in values before and after PR divided by the mean SD (ie, mean SD at baseline and follow-up). It therefore expresses the change in response to treatment against the variability of the parameter in the population being studied Data are presented as mean $\pm S D$ unless otherwise specified, and a $p$ value of $<0.05$ is taken as significant.

\section{RESULTS}

Data on CAT score before and after pulmonary rehabilitation were available for 297 individuals, age $69.2 \pm 9.3$ years, $62.7 \%$ male, $\mathrm{FEV}_{1} 50.9 \pm 18.9 \%$ predicted, MRC dyspnoea score 
Table 1 Participating centres and outcome measures employed

\begin{tabular}{|c|c|c|}
\hline Name of participating centre & $\begin{array}{l}\text { No. of } \\
\text { patients }\end{array}$ & Outcome measures \\
\hline $\begin{array}{l}\text { Royal Brompton and Harefield Foundation } \\
\text { NHS Trust }\end{array}$ & 87 & $\begin{array}{l}\text { SGRQ, ISWT, ESWT, HAD, } \\
\text { MRC, CRO }\end{array}$ \\
\hline St George's Hospital NHS Trust & 22 & ISWT, SGRO \\
\hline $\begin{array}{l}\text { Kings Health Partners/Guy's and St Thomas' } \\
\text { Foundation NHS Trust }\end{array}$ & 66 & $\begin{array}{l}\text { 6MWT, ISWT, CRO, HAD, } \\
\text { MRC }\end{array}$ \\
\hline Greenwich PCT & 57 & 6MWT, CCQ \\
\hline Kensington and Chelsea PCT & 11 & ISWT, ESWT, CRO, HAD \\
\hline Croydon PCT & 34 & ISWT, CRO, HAD \\
\hline Westminster PCT & 17 & ESWT, CRO, HAD, MRC \\
\hline
\end{tabular}

CCQ, Clinical COPD (chronic obstructive pulmonary disease) Questionnaire; CRO, Chronic Respiratory Questionnaire; ESWT, endurance shuttle walk test; HAD, Hospital Anxiety and Depression score; ISWT, incremental shuttle walk test; MRC, Medical Research Council dyspnoea score; 6MWT, 6 min walk test; PCT Primary Care Trust; SGRQ, St George's

Respiratory Questionnaire.

3.4 \pm 1.0 , median body mass index (BMI) 27.7 \pm 6.5 . In response to the anchor question, 162 reported that they were much better after PR, 88 a little better, 8 no different and 3 a little worse. In 36 cases the anchor question was not completed. The group in whom the anchor question was not documented did not differ significantly from those in whom it had been recorded. Although not therefore available for the primary outcome, data from these 36 cases were retained for other analyses. Location, recruitment figures and outcome measures used at different sites are given in table 1 .

Baseline CAT score correlated in univariate analysis with other health status measures, MRC score, ISWT distance, age and airflow obstruction, as expected, with a higher CAT score associated with worse breathlessness, anxiety, depression and functional exercise capacity (table 2). The CAT score was lower in men, $19.7 \pm 7.2$ versus $21.5 \pm 7.5(p=0.03)$. In a stepwise regression analysis including age, MRC dyspnoea score, BMI, gender and $\mathrm{FEV}_{1} \%$ predicted ( $\mathrm{n}=178$ with these data available), only age and MRC dyspnoea score were retained, giving the equation; CAT score $=18.8+3.9$ (MRC score) -0.16 (age) $\left(\mathrm{r}^{2}=0.29\right)$.

The response of the various outcome measures to rehabilitation are given in table 3 together with estimation of the effect size. The CAT improved significantly following rehabilitation, $-2.9 \pm 5.6(p<0.001)$, as did all other measures of health status and functional capacity. The effect size of rehabilitation on CAT score was moderate $(\mathrm{d}=0.4)$; other health status measure effect sizes were variable (CCQ . .6, CRQ 0.8 and SGRQ 0.2), although

Table 2 Univariate correlates of baseline CAT score

\begin{tabular}{lccc}
\hline Variable & $\begin{array}{c}\text { Correlation } \\
\text { coefficient }\end{array}$ & $\begin{array}{l}\text { No. of } \\
\text { observations }\end{array}$ & p Value \\
\hline Age & 0.24 & 296 & $<0.001$ \\
FEV $_{1}$ \% predicted & -0.17 & 239 & 0.008 \\
MRC dyspnoea score & 0.44 & 252 & $<0.001$ \\
HAD anxiety & 0.37 & 215 & $<0.001$ \\
HAD depression & 0.36 & 215 & $<0.001$ \\
CCQ & 0.68 & 58 & $<0.001$ \\
CRQ total & -0.33 & 297 & $<0.001$ \\
SGRO total & 0.74 & 38 & $<0.001$ \\
ISWT & -0.36 & 211 & $<0.001$ \\
GMWT & -0.27 & 68 & 0.03 \\
InESWT & -0.28 & 38 & 0.084
\end{tabular}

CAT, COPD assessment test; CCQ, Clinical COPD Questionnaire; COPD, chronic obstructive pulmonary disease; $C R Q$, chronic respiratory questionnaire; $\mathrm{FEV}_{1}$ forced expiratory volume in $1 \mathrm{~s}$; HAD, Hospital Anxiety and Depression score; ISWT, incremental shuttle walk test; InESWT, log-transformed endurance shuttle walk test; MRC, Medical Research Council dyspnoea score; $6 \mathrm{MWT}, 6$ min walk test; SGRQ, St George's Respiratory Questionnaire.
Table 3 Response to pulmonary rehabilitation (PR)

\begin{tabular}{lccccc}
\hline & Before PR & After PR & Change & p Value & $\begin{array}{c}\text { sffect } \\
\text { size (d) }\end{array}$ \\
\hline CAT $(n=297)$ & $20.5 \pm 7.4$ & $17.5 \pm 7.7$ & $-2.9 \pm 5.6$ & $<0.001$ & 0.4 \\
HAD anxiety $(n=211)$ & $7.3 \pm 4.2$ & $6.1 \pm 4.1$ & $-1.3 \pm 3.3$ & $<0.001$ & 0.3 \\
HAD depression ( $n=211)$ & $6.7 \pm 3.6$ & $5.2 \pm 3.2$ & $-1.5 \pm 3.0$ & $<0.001$ & 0.4 \\
CRO total $(n=195)$ & $14.9 \pm 4.1$ & $18.2 \pm 4.2$ & $3.3 \pm 3.8$ & $<0.001$ & -0.8 \\
ISWT (m) ( $n=191)$ & $238 \pm 148$ & $309 \pm 175$ & $70 \pm 83$ & $<0.001$ & -0.4 \\
MRC dyspnoea score & $3.4 \pm 1.0$ & $2.9 \pm 1.1$ & $-0.6 \pm 0.7$ & $<0.001$ & 0.6 \\
(n=130) & & & & & \\
6MWT (m) ( $n=68)$ & $267 \pm 94$ & $339 \pm 105$ & $72 \pm 74$ & $<0.001$ & -0.7 \\
CCO (n=57) & $3.0 \pm 1.2$ & $2.3 \pm 1.3$ & $-0.7 \pm 0.9$ & $<0.001$ & 0.6 \\
SGRO total (n=39) & $45.7 \pm 19.4$ & $41.8 \pm 17.2$ & $-3.9 \pm 9.0$ & $<0.001$ & 0.2 \\
ESWT (s) (n=36) & $294 \pm 215$ & $502 \pm 393$ & $208 \pm 47$ & 0.001 & -0.7 \\
\hline
\end{tabular}

Measures are presented in order of frequency with which they were measured.

$p$ values are for paired t tests.

CAT, COPD assessment test; CCQ, Clinical COPD Questionnaire; COPD, chronic obstructive pulmonary disease; $\mathrm{CRO}$, chronic respiratory questionnaire; ESWT, endurance shuttle walk test; HAD, Hospital Anxiety and Depression score; ISWT, incremental shuttle walk test; MRC, Medical Research Council dyspnoea score; 6MWT, 6 min walk test; SGRO, St George's Respiratory Questionnaire.

the numbers completing each measure were variable-for example, the SGRQ was used in only 39 participants, which means that direct comparisons require caution.

Change in CAT score in response to rehabilitation was independently associated with baseline CAT score, falling most in those with the highest baseline symptom burden ( $\mathrm{r}=-0.34$ $\mathrm{p}<0.0001)$. The score also improved more in women: $\Delta$ CAT $-3.8 \pm 6.1$ versus $-2.2 \pm 5.3(\mathrm{p}=0.019)$, but was not associated with baseline dyspnoea, anxiety and depression, walking distance or airflow obstruction.

Change in CAT score following PR correlated significantly with changes in other measures of response to PR (table 4). Figure 1 shows the correlation between the change in the most frequently recorded measure of health status (the CRO total score, $\mathrm{n}=195)$ and $\Delta$ CAT score following PR.

The data in table 5 show the change in CAT and other outcome measures following PR according to the different anchor response categories. Change in CAT score was sensitive to different levels of response to the anchor question ( $-3.8 \pm 6.1$ 'much better'; $-1.3 \pm 4.5$ 'a little better', $\mathrm{p}=0.002$ ) (figure 2). Only small numbers were available for the response categories 'no different', $\mathrm{n}=8 \Delta \mathrm{CAT}-2.3 \pm 3.3$; and 'a little worse', $\mathrm{n}=3$ $\Delta$ CAT $+2 \pm 0$.

Table 5 shows that in addition to change in CAT score, change in $\mathrm{CRO}$ and the Hospital Anxiety and Depression (HAD) scores also differed significantly across anchor responses.

Table 4 Univariate correlates of change in CAT score

\begin{tabular}{lccc}
\hline Variable & $\begin{array}{l}\text { Correlation } \\
\text { coefficient }\end{array}$ & $\begin{array}{l}\text { No. of } \\
\text { observations }\end{array}$ & p Value \\
\hline$\Delta$ CRO total & -0.41 & 195 & $<0.001$ \\
$\Delta$ SGRO total & 0.36 & 38 & 0.03 \\
$\Delta C C Q$ & 0.13 & 57 & 0.034 \\
$\Delta$ HAD anxiety & 0.15 & 210 & 0.03 \\
$\Delta$ HAD depression & 0.17 & 211 & 0.01 \\
$\Delta$ ISWT & -0.19 & 191 & 0.008 \\
$\Delta$ GMWT & 0.31 & 68 & 0.01 \\
$\Delta$ InESWT & -0.19 & 27 & 0.35 \\
$\Delta$ MRC & 0.20 & 130 & 0.02
\end{tabular}

CAT, COPD assessment test; CCQ, Clinical COPD Questionnaire; COPD, chronic obstructive pulmonary disease; CRO, chronic respiratory questionnaire; HAD, Hospital Anxiety and Depression score; ISWT, incremental shuttle walk test; InESWT, log-transformed endurance shuttle walk test; MRC, Medical Research Council dyspnoea score; $6 \mathrm{MWT}, 6$ min walk test; SGRO, St George's Respiratory Questionnaire. 


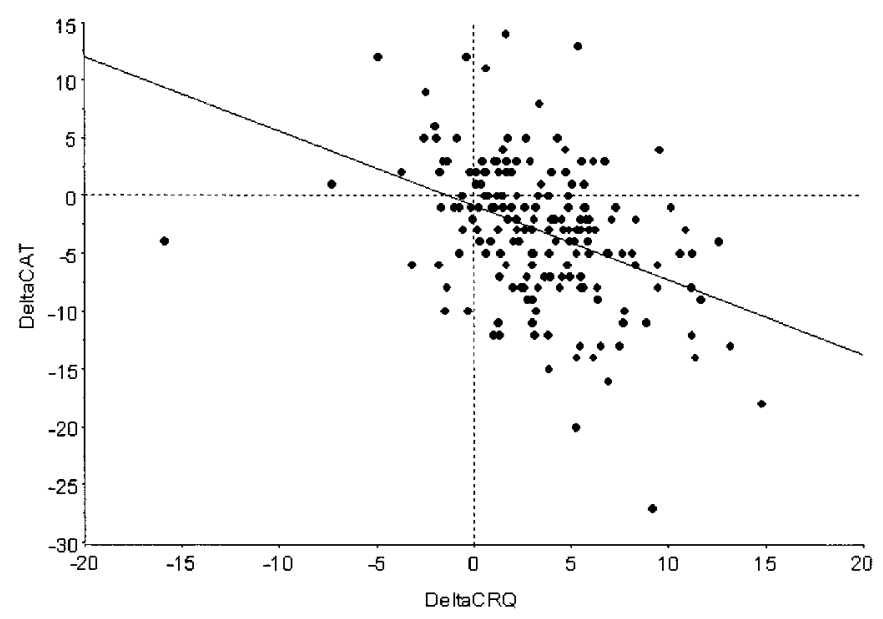

Figure 1 Change in COPD (chronic obstructive pulmonary disease) assessment test (CAT) in response to pulmonary rehabilitation correlated with change in Chronic Respiratory Questionnaire (CRO) total score $(r=-0.41 ; p<0.001)$.

\section{DISCUSSION}

The main findings of the present study are that the CAT score is responsive to $\mathrm{PR}$ and can discriminate between different levels of subjective response when compared with an anchor question, with a fall of 1.3 points corresponding to 'a little better' and 3.8 points to 'much better'. It correlated with improvements in other outcome measures, and the estimated effect size of PR on change in CAT was moderate.

\section{Methodological issues}

A strength of this study is that it included a relatively large sample of unselected patients taking part in clinical PR programmes with data collected prospectively across multiple sites. As such, it is likely to be generalisable to routine clinical practice. The centres participating added the CAT score alongside the data they were already collecting routinely, which meant that not all patients had all outcomes measured. Caution is therefore needed in comparing the different measures, as sample sizes available for the comparisons vary. In particular, the sample size for the SGRQ is small.

The MCID for the CAT has not yet been established. Based on a mapping exercise with the published data comparing the CAT and SGRQ, ${ }^{7}$ the 4-unit MCID for the SGRQ corresponds to a value of 1.6 units for the CAT MCID. An objective of this study was to provide further data that might contribute to the CAT MCID estimation process, but the effectiveness of PR meant that the full range of the anchor question responses $1-5$ was not used. This type of one-direction change limits the reliability of MCID estimates; however, the change of 1.3 units observed in the 53 patients who reported that they were 'a little better' is of a similar magnitude to the change in CAT score that corresponds to the SGRQ MCID. An alternative approach is to use the MCID of another validated and repeatable clinical measure such as the CRQ as an anchor for those that respond to $\mathrm{PR}$. This technique has been used previously when estimating MCID for exercise tests in COPD. ${ }^{16}$ However the use of linear regression in this way assumes no measurement error ${ }^{17}$ and its reliability is dependent upon the strength of correlation between the two measurements, which in the case of the CRQ was only 0.4 (figure 1).

\section{Significance of the findings}

The data suggest that the CAT score can be used as an outcome measure in patients with COPD taking part in PR. Since the tool is quick to complete and score and can therefore be integrated into routine clinical practice, it offers the possibility of a closer integration between PR and other aspects of care. The CAT can be completed by patients online, which may be of use in the home management and maintenance of rehabilitation. We acknowledge that certain tools used for baseline assessment may provide specific information that will influence clinical practice, for example the HAD score may guide referral for psychological support, and the lung information needs questionnaire (LINQ) might identify individuals who need particular attention to their

Table 5 Change in outcome measure by anchor question response

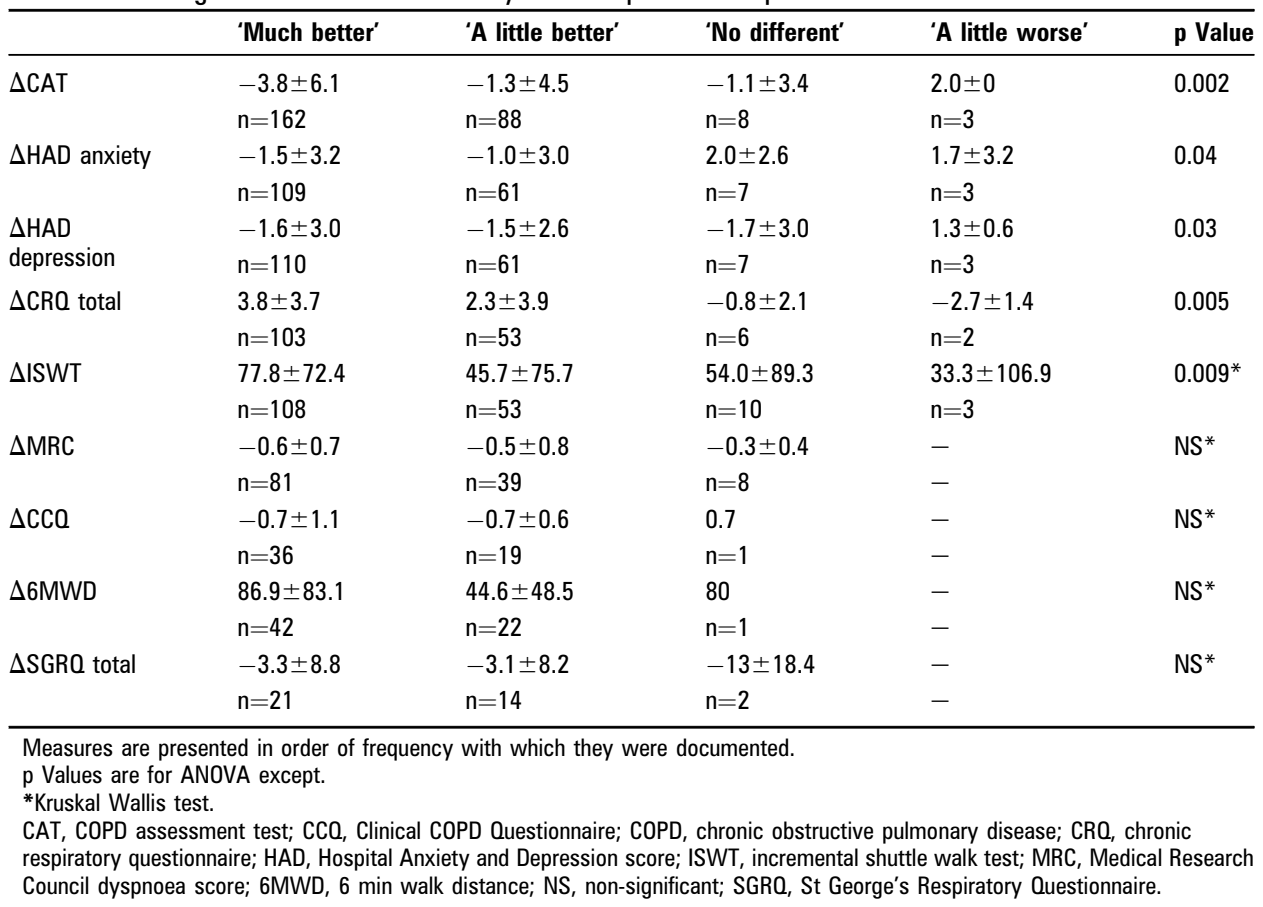




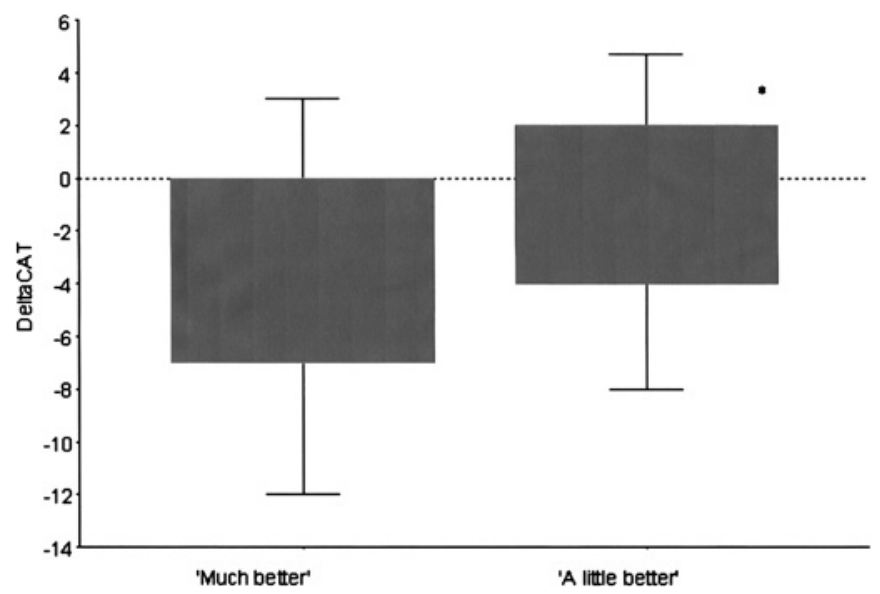

Figure 2 Change in COPD (chronic obstructive pulmonary disease) assessment test (CAT) score after pulmonary rehabilitation corresponding to different responses to the anchor question 'How do you feel your overall condition has changed after rehabilitation?': 'much better' $\mathrm{n}=162, \Delta$ CAT $-3.8 \pm 6.1$; ' a little better' $\mathrm{n}=88, \Delta$ CAT $-1.3 \pm 4.5$ $(p<0.002)$. As only small numbers were available for these categories, data for 'no different' $\mathrm{n}=8 \Delta$ CAT $-2.3 \pm 3.3$; and 'a little worse' $\mathrm{n}=3$ $\Delta$ CAT $+2 \pm 0$ are not shown.

understanding of their disease. ${ }^{18} 19$ A significant amount of time and effort is required by both patients and health professionals in the completion and scoring of various outcome measures around PR, which has an opportunity cost for the delivery of the PR intervention itself. Given the resource limitations that exist, the widespread introduction of a simpler tool such as the CAT may have significant cost benefits.

An important observation is that the overall effectiveness of $\mathrm{PR}$ in the programmes in this study was similar to that outlined in a recent meta-analysis of clinical trials of $\mathrm{PR}$, where improvements in CRQ (0.77-1.1) and SGRQ (-6.1) exceeded their respective MCIDs. ${ }^{2}$ This suggests that outcomes achieved in routine clinical practice are comparable with those observed in clinical trials, at least in patients completing the programme. In our study the participants exceeded the MCID for the CRO, the 6MWT and the ISWT (table 3). ${ }^{10} 1216$ In fact the effectiveness of PR meant that the full range of the anchor question responses 1-5 was not used-only 8 (3\%) subjects reported no improvement and $3(1 \%)$ that they were a little worse. This may appear to differ from the results of Singh et al who found a wider range of responses when they assessed the MCID for the ISWT, but it should be noted that the anchor question in that study addressed exercise capacity specifically rather than the more general question in the present paper 'How do you feel your overall condition has changed after rehabilitation?'. ${ }^{12}$

\section{Conclusion}

The CAT score is responsive to $\mathrm{PR}$ and able to distinguish different levels of response. Widespread adoption of the CAT as a substitute for more time-consuming questionnaires has the potential to streamline PR provision and improve benchmarking between programmes.

Acknowledgements The authors would like to acknowledge the following for their assistance in collecting data: Karen Ingram, Ria Fowler and Amy Clark at Harefield Hospital; and Hellene Bellas at King's College Hospital.

Funding The National Institute of Health Research (NIHR) Respiratory Biomedical Research Unit Royal Brompton \& Harefield NHS Foundation Trust and Imperial College. WD-CM is funded by an NIHR Clinician Scientist award. The views expressed in this publication are those of the authors and not necessarily those of the NHS, the NIHR or the Department of Health. NSH is an HEFCE Clinical Senior Lecturer.

\section{Competing interests None.}

Ethics approval This study was conducted with the approval of the Riverside Research Ethics Committee.

Provenance and peer review Not commissioned; externally peer reviewed.

\section{REFERENCES}

1. Nici L, Donner C, Wouters E, et al. American Thoracic Society/European Respiratory Society Statement on Pulmonary Rehabilitation. Am J Respir Crit Care Med 2006:173:1390-413.

2. Lacasse $\mathbf{Y}$, Goldstein R, Lasserson TJ, et al. Pulmonary rehabilitation for chronic obstructive pulmonary disease. Cochrane Database Syst Rev 2006;(4):CD003793.

3. Seymour JM, Moore L, Jolley CJ, et al. Outpatient pulmonary rehabilitation following acute exacerbations of COPD. Thorax 2010;65:423-8.

4. Jones PW, Quirk FH, Baveystock CM, et al. A self-complete measure of health status for chronic airflow limitation. The St. George's Respiratory Questionnaire. Am Rev Respir Dis 1992;145:1321-7.

5. Guyatt GH, Berman LB, Townsend M, et al. A measure of quality of life for clinical trials in chronic lung disease. Thorax 1987;42:773-8.

6. van der Molen T, Willemse BW, Schokker S, et al. Development, validity and responsiveness of the Clinical COPD Questionnaire. Health Qual Life Outcomes 2003;1:13.

7. Jones PW, Harding G, Berry $P$, et al. Development and first validation of the COPD Assessment Test. Eur Respir J 2009;34:648-54.

8. CAT Development Steering Group. COPD assessment test-healthcare professional user guide. http://www.catestonline.org/images/UserGuides/ CATHCPUser\%20guideEn.pdf (accessed 31 Jan 2011)

9. Kocks JW, Tuinenga MG, Uil SM, et al. Health status measurement in COPD: the minimal clinically important difference of the clinical COPD questionnaire. Respir Res 2006; 7:62.

10. Schunemann HJ, Puhan M, Goldstein R, et al. Measurement properties and interpretability of the Chronic respiratory disease questionnaire (CRO). COPD 2005;2:81-9.

11. Singh SJ, Morgan MD, Scott S, et al. Development of a shuttle walking test of disability in patients with chronic airways obstruction. Thorax 1992;47:1019-24.

12. Singh SJ, Jones PW, Evans R, et al. Minimum clinically important improvement for the incremental shuttle walking test. Thorax 2008;63:775-7.

13. Revill SM, Morgan MD, Singh SJ, et al. The endurance shuttle walk: a new field test for the assessment of endurance capacity in chronic obstructive pulmonary disease. Thorax 1999;54:213-22.

14. American Thoracic Society. ATS statement: guidelines for the six-minute walk test. Am J Respir Crit Care Med 2002;166:111-17.

15. Casanova C, Celli BR, Barria P, et al. The 6 min walk distance in healthy subjects: reference standards from seven countries. Eur Respir J 2010;37:150-6.

16. Puhan MA, Chandra D, Mosenifar Z, et al. The minimal important difference of exercise tests in severe COPD. Eur Respir J 2011;37:784-90.

17. Dodd JW, Jones PW. Limitations of calculating 'true' regression slope: impact on estimates of MID. Eur Respir J 2011. In Press.

18. Jones RC, Wang $X$, Harding $S$, et al. Educational impact of pulmonary rehabilitation: lung Information Needs Questionnaire. Respir Med 2008; 102:1439-45

19. Hyland ME, Jones RC, Hanney KE. The lung information needs questionnaire: development, preliminary validation and findings. Respir Med 2006;100:1807-16. 